\title{
Index of names and subjects
}

A

abolitionist 242, 243

Adenauer, Konrad 89, 90, 91, 114

Ahonen, Risto 177

Al-Andalus 55, 213

alliance of throne and altar 254

Altermatt, Urs 47, 48, 49, 90, 210,

227, 230, 239

American Revolution 240

Anabaptists 64, 256

anti-Judaism 62, 245, 246

antisemitism 73, 135, 160, 175, 245

assimilation 162, 163, 165, 171, 218

atheism 17, 264, 265

B

Bach, Johann Sebastian 230

Bacon, Francis 54

Bakke, Elisabeth 126, 127, 128, 129, 130

Barbarians 19, 22, 44, 150, 205

Barraclough, Geoffrey 99

Barroso, José-Manuel 75, 117

Bauman, Zygmunt 77

Beethoven, Ludwig van 123

behaving without belonging 279 ,

290, 291, 292

believing without belonging 279 ,

290, 291, 293

Benelux 92

Berger, Peter 270

Berlinski, Claire 252
Berlin Wall 48, 93, 114

Béthune, Maximilien de 85

bicultural communities 159

Billings, Allan 269, 270, 291, 292

birth rates 25

Blair, Tony 232

Boerwinkel, Feitse 254, 255, 258

Bosch, David 274

Brandt, Willy 83

Broggini, Jérôme 53, 204, 206

Brown, David 233

Bruguès, Jean-Louis 227, 228

Buchman, Frank 89

Buijs, Govert 229

Bureau of European Policy Advisors 117

Burke, Edmund 66, 67

Bush, George senior 96

Byzantine Empire 58

Byzantine 'Europe' 59

C

Caldwell, Christopher 169

capitalism 63, 105, 209, 237, 244, 245

Champion's League 149

Charlemagne $57,58,84,88,128$,

129, 236

Charter of Fundamental Rights 131

Cherniae, Anatoli 96

China 134

Chopin, Thierry 31, 186

Christendom 58, 60, 62, 63, 81, 124 , 206, 211, 224, 253, 254, 258, 274, 316 
Christian democratic politicians 88

Christian mission 70

Christian realm, Europe as 54, 55, 58,

$59,60,62,63,74,213,218$

Churchill, Winston 47,87

civilization offensive 69

Clovis 54,57

Cold War 73, 80, 87, 98, 99, 115, 134 colonialism $33,36,69,73,225,237$,

240,242

common roots 131

common values 130

complementary identities 130

Constantine 58, 59, 253, 254, 255

Constantinian system 255, 256

Constantinople 50, 55, 58, 59, 60,

254

corpus christianum 58, 64, 225, 239

Coudenhove-Kalergi, Richard 86

Council of Europe 87

Courbage, Youssef 171

Crusades 59, 61, 213, 236, 277

cuius regio, eius religio 62

cultural capitals of Europe 121

cultural European identity 131

\section{D}

Dalarun, Jacques 237, 238

Davie, Grace 270, 271, 290, 291,

293, 294, 299, 300

Day of Europe 91, 123

Declaration on European Identity

(1972) 119

default religion 294

De Gasperi, Alcide 89, 90, 91, 114,

184

Delgado, Mariano 210, 229, 230,

238, 239

Delors, Jacques 116, 181, 198, 199

Democratic deficit 185

Derrida, Jacques 267

Dialogue of EU with Churches and

other faith communities 116, 199

Dias, Bartholomeas de 241

Diaspora communities 167

Diprose, Ronaldo 287

Dunant, Henri 83
E

Eco, Umberto $38,122,148$

Edict of Milan (313) 253, 254

Enlightenment $65,66,67,68,69$, $77,84,135,137,203,208,209,210$ $216,238,242,247,256,257,263$.

$266,267,276$

Erasmus 61, 147, 209

Erasmus program 122

etsi deus non daretur 261

Eurabia 177

Eurasianism 105

Euratom 91, 92

euro $31,101,123,133,182,187$

Eurocentrism 36, 107, 111, 139, 191, 236

Euro-consciousness 132

euro-crisis 122,183

European Charter of Fundamental

Rights 75

European Community 92

European Community of Coal and

Steal 91

European Constitution, project 184,

215

European Convention of Human

Rights 87, 92, 113

European Court of Human Rights 92,

102

European driver's license 123

European Economic Community 91

European 'elite' 126

European Evangelical Alliance 137

European flag 117, 123

European Fund of Regional Develop-

ment (EFRED) 92

European identity 127, 136

Europeanism 126

Europeanization 70, 119, 121, 149,

177

Europeanness 75, 79, 130

European Parliament 38, 92, 100,

102, 107, 108, 109, 123, 182, 186,

201, 219

European passport 123

European Values Studies 290 
,Europe' as a cultural zone 77

'Europe' as a family of cultures. 77

'Europe' as a house of nations 79, 80

'Europe' as a revived Roman Empire 110

'Europe' as civilization 65

Europé, name, myth 19, 20, 22

Euro-scepticism 119, 191

Eurostat 156

Evangelical European Alliance 111

exceptional continent 270

\section{F}

Ferry, Luc 275, 276

Fokas, Effie 270

Fontaine, Pascal 32, 130, 131

Fountain, Jeff 90

Frankish (Carolingian) kingdom 57,

129

freedom of religion $66,67,116,266$

French Revolution 208, 209, 210,

$215,217,238,239,240,243,256$,

258,263

\section{G}

Gantenbein, Hansjörg 274

García, Soledad 131, 132

Gauchet, Marcel 196

Gaulle, General de 79,93

Giuliani, Jean-Dominique 187

glasnost 94

globalisation 167

globalization $30,31,45,77,125$,

$187,188,189,191,221,315$

Goethe, Wolfgang 205

Gorbachev 95

Gorbachev, Mikhail 80, 93, 94, 96,

97, 98, 99

Goya 233

Graceffa, Agnès 57

Gulag Archipelago 267

\section{H}

Habermas, Jürgen 184, 185, 218 ,

219, 267

Habsburg 84, 129

Heitz, Arsène 117
Herodotus 20

Hervieu-Léger, Danièle 293, 298

Hobbes, Thomas 77

Holy Roman Empire 57

Hugo, Victor 86, 133

Human and Citizens Rights 240

Humanism 210, 216, 239

human rights $67,75,76,80,84,87$,

$100,107,114,119,130,131,155$,

$172,188,189,190,191,193,199$,

208, 211, 218, 223, 227, 229

Humphrey, Caroline 105

Huntingdon, Samuel 81

I

immigration, external 154, 156

immigration, issue of 165

immigration of Christians 158

immigration policy 110,111

immigration surplus 25

Industrial Revolution 210, 244, 245

Ingleby, Jonathan 167, 315

integration $13,16,26,30,33,38,45$, $46,88,90,92,106,111,112,116$, $119,120,121,122,127,129,142$,

$148,150,153,157,162,163,165$.

$174,177,182,187,188,190,191$,

$192,195,197,198$

Iron Curtain 87, 88, 93, 114, 115

Islamic extremism 174

Islamization 161

Islam, radical forms 160, 173, 174, 177

Islam, traditional forms 173

\section{]}

Jackson, Darrell 136, 315

Jacques, Francis 221

Jeltsin, Boris 95

Jenkins, Philip 158, 162, 171, 177 .

$219,252,271,286,300$

John Paul II 94, 207, 211, 217

K

Kant, Immanuel 68, 77, 85, 209

Kasper, Walter 114, 208, 236

King, Martin Luther 83, 244 
Kohl, Helmut 96, 97, 98, 99

Kundera, Milan 49

Kuyper, Abraham 245

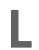

Lactantius 21

laïcité 75, 257, 276

Lausanne Committee of World Evan-

gelisation 289

Leitkultur 157, 160, 163, 284

Lewis, David Levering 55, 57, 60, 213

life expectancy 25

Livi-Bacci, Massimo 315

Livingstone, David 70

Llul, Raymond 213

Locke, John 68

Longman, Philip 28

Lorein, Johan 260, 261

Lumengo, Ricardo 163, 165

Lustiger, Jean-Marie 235, 236

Luther, Martin 64

Lyotard, Jean-François 267

\section{M}

marginal Church membership 286,

288

Martel, Charles 57

Martin, Catherine 50, 51

Massignon, Bérengère 197, 199

Merkel, Angela 94, 162, 182, 196

Micklethwait, John 170, 298, 300

Middle-Ages 22, 46, 47, 57, 60, 62,

64, 217, 253

migrant workers 154

Milit 214

minimal Church membership 287

Monnet, Jean 91

Moors 55, 212, 213

More, Thomas 61

mosaic model 161

Müller, Jan-Werner 194

multiculturalism 161, 162, 163, 168,

217,218

multicultural society $16,131,134$, $153,157,159,161,163,167,173$. $174,178,188,217,218,268,277$. 284,293 multilingual 148

multireligious society $12,163,284$,

295

Murray, Stuart 274

Muslim presence in Europe 170, 172

Muslims in Europe 42

Muslims in Europe; demographic

growth prospects 171

Muslim world 30, 55, 58, 61, 64, 81,

$82,134,135,212,214$

\section{N}

Napoleon 84, 129

nationalism 124

national myth 199, 218

nation and its (imagined) past 125

neo-paganism 297

Newbigin, Lesslie 12, 273, 274, 280,

$281,300,301,316$

Ni Dieu, ni maître 239

Nobel, Alfred 83

Nobel Peace Prize 83, 84

nominal Church members 286

Non-Conformists 64, 256

0

ora et labora 115

Orban, Viktor 75

Orthodox Europe 40

Ottoman Empire 61

P

Pacensis, Isidorus 55

Pagden, Anthony 49, 53, 54, 65, 70,

74, 78, 123, 208

Parliamentary democracy 130

Pascal, Blaise 252

patriotism 119, 124, 134, 138, 149 ,

$164,191,218$

Penn, William 85

perestroika 94

pluralism 75, 257

Podiebrad, Georges 85

Poitiers, Battle of 55

population 24 
population decrease 26

populist movements and parties 16 ,

164, 191, 192, 217

post-Christendom 253

post-Christian 17, 251, 252, 261,

267, 275, 284

post-Christianised 251, 253

post-colonialism 154

post-Constantinian 253

post-evangelised 268

postmodernism 216, 267, 268

post-religious 261

post-secular 297, 300

Poupard, Paul 205, 206

Prodi, Romano 117

progress, idea of 68

Protestant Europe 40, 62

Putin, Vladimir 104

R

radical Islam 174

Raiffeissen 245

Rapid Deployment Force 188, 189

Ratzinger, Joseph 53, 67, 168, 217 ,

220, 314

Ratzinger, Joseph (Pope Benedict VIV)

$53,67,167,217$

Ratzinger, Joseph (Pope Benedict XVI)

53, 220

reconciliation (between former en-

emies) 88

reconquista 60,213

Reformation 62, 64, 66, 209, 253,

266

Rembrandt 233

Renaissance 33, 66, 204, 209, 216

Rerum Novarum 89, 245

Reynold, George de 21

Rietkerk, Wim 219, 220, 226, 227.

$236,237,247,248$

Rifkin, Jeremy 108

Ripa, Cesare 65

Roberts, Dana 241, 242

Roman Catholic Europe 40

Roman Empire 57, 59, 245, 253, 255 roots of Europe, Christian 11, 16, 114, 131, 136, 162, 197, 203, 204, 205, 208, 209, 211, 212, 214, 215 , 216, 217, 218, 219, 221, 227, 246 roots of Europe, Greek 204 roots of Europe, idealised Christian 216

roots of Europe, Jewish 206, 211

roots of Europe, Muslim 212

roots of Europe, Roman 204

roots of Europe, Slavic 207

Rousseau, Jean-Jacques 85

Ruler, Anton van 247

$\mathbf{S}$

Santer, Jacques 117

Sarracenes 55

Schaeffer, Francis 248

Schengen Agreement 100, 135, 136, 164,188

Schirrmacher, Christine 177, 178

Schmidt, Helmut 195

Schuman Declaration 91, 123

Schuman, Robert 11, 89, 90, 91, 114 , $186,189,314,315$

Second World War 154, 247

secularisation 166, 190, 251, 257 , 263, 264, 265, 266, 270, 271, 275 ,

276, 279, 291, 295, 297, 299, 300

Secularist agenda 190, 216

seekers of asylum 155

separation between ethnic communi-

ties 159

separation of Church and State 119 ,

$130,256,257$

Seye, Boucounta 21

Sharia $173,177,178$

Shoa 236, 246, 267

Slavery 242, 244

Smith, Anthony $66,69,124,125$, $126,132,134,135,137,149$

Smith, Calvin 79, 80, 111

socio-cultural cross 43, 46

soul of/for Europe 198 
Soviet Union, dissolution 95 stadial theory of history 68 Stakos, Metropolitan Mikhail 80 Stalin, Joseph 47,87

State Church 255, 256, 257, 258

Sticht, Pamela 78, 120, 203, 204, 205

Strahlenberg 22

Susbeille, Jean-François 118, 296

Szczypiorski, Andrzej 48

Szücs, Jenö 48, 49

\section{T}

Tabbert, Philipp Johann 22

Taizé 297

Thatcher, Margaret 96

Theodosius 255

Theresa, Mother 83

Treaty of Amsterdam (1997) 100

Treaty of Lisbon (2008) 75, 101, 117. $131,182,185,190,215$

Treaty of London (1949) 87

Treaty of Maastricht (1991) 100, 101, $122,123,134,182,198$

Treaty of Paris (1951) 91

Treaty of Rome (1957) 91

Treaty of Utrecht (1713) 62

Treaty of Verdun (843) 57

Treaty of Westphalia (1648) 62

Tully, James 68

Turkish membership 104

U

ummah 177

United States of Europe 86, 120

unity in diversity 107, 120, 138

V

Valéry, Paul 204, 205

Van Gogh 233

Verhofstadt, Guy 183

vestiges 259, 261

vicarious religion 293

Vives, Juan Luis 61

\section{W}

Walldorf, Friedemann 12, 274

Weber, Max 63, 209

Weigel, George 197, 236

Weiler, Joseph 194

Wende 93

Western civilization 81

Wheat and tares, Parable of 248

Wilberforce, William 243, 244

Woolbridge, Adrian 170, 298, 300

Y

Yalta 47,87

Yugoslavia, war in former $42,75,76$

Z

Zapek, Karel 181 